\title{
AN AUTOMATIC SEARCH FOR FLARE STARS IN SOUTHERN STELLAR AGGREGATES OF DIFFERENT AGES*
}

\author{
R. ANIOL, H. W. DUERBECK and W. C. SEITTER \\ Astronomical Institute of Muenster University, F.R.Germany \\ M. K. TSVETKOV 1 \\ Department of Astronomy, Academy of Sciences, Sofia, Bulgaria \\ and Astronomical Institute of Muenster University, F.R.Germany
}

\begin{abstract}
Statistically relevant samples of flare stars in stellar aggregates can be used to specify the stellar mass-age-activity-relation when the ages of the aggregates are known from independent investigations. Associations and clusters of the southern sky are currently surveyed with the GPO astrograph of the European Southern Observatory. The plates are digitized with the PDS $2020 \mathrm{GM}^{\text {plus }}$ microdensitometer and the data are reduced automatically at the Astronomical Institute of Muenster University. The programme package "FLARE" is described. First results from the Orion association are presented.
\end{abstract}

\section{Introduction}

Stellar activity as observed in flare stars (UV Cet stars) and related objects (T Tau, H $\alpha$ emission, FU Ori stars) appears to play an important role during the early stages of stellar evolution (Ambartsumian 1969). The underlying physical processes and their decline with advancing age are, however, still not sufficiently well understood. Thus, it is of interest to study not only individual objects in detail but to improve the statistics of active stars and their degree of activity. The project described here aims at enlarging the number of known flare stars (currently 1414 verified plus 188 suspected UV Cet-type variables), and, in particular, to derive a mass-age-activity-relation from the frequency of flares and flare stars of different masses in stellar aggregates of various ages.

In addition to the general need for more data, the clear dichotomy of the data available in the northern and southern hemispheres requires additional surveys particularly in the south. The status of observations prior to the present study is shown in Tables 1 and 2 and in Fig. 1.

\section{The Muenster Flare Star Project}

The Muenster Flare Star Project started in 1985. The large demand on southern Schmidt telescope time for other surveys and atlases prompted us to work with the GPO astrograph of the European Southern Observatory, hoping that the smaller flare amplitudes in a blue band which does not extend into the ultraviolet would be compensated by the faster growth

\footnotetext{
*This paper, based on observations collected at the European Southern Observatory, La Silla, Chile, is dedicated to Academician V.A. Ambartsumian in honour of the 80th anniversary of his birthday.

${ }^{1}$ Research fellow of the Alexander von Humboldt-Stiftung Bonn-Bad Godesberg.
} 
TABLE 1. Flare data in northern stellar aggregates.

\begin{tabular}{l|ccl}
\hline \multicolumn{1}{c|}{ aggregate } & $\begin{array}{c}\text { no. of } \\
\text { flare stars }\end{array}$ & $\begin{array}{c}\text { total observing } \\
\text { time (h) }\end{array}$ & references \\
\hline Pleiades & 539 & 3250 & $1,2,3$ \\
Orion M42/M43 & 499 & 1590 & $4,5,6,7$ \\
Taurus Dark Clouds & 102 & 870 & 8 \\
NGC 7000-IC5068-70 & 75 & 1185 & 9 \\
Praesepe & 54 & 660 & $10,11,12$ \\
Monoceros - NGC 2264 & 42 & 100 & 13 \\
Cassiopeia-W3, IC 1848 & 25 & 62 & $14,15,16,17$ \\
$\gamma$ Cyg-IC 1318 & 17 & 300 & 9 \\
Coma Open cluster & 14 & 338 & 18 \\
Cepheus-NGC 7023 & 14 & 50 & 19,20 \\
\hline total & 1381 & 8404 & \\
\hline
\end{tabular}

(1) Haro et al. 1982; (2) Chavushian 1979; (3) Tsvetkov et al. 1989; (4) Haro 1953; (5) Natsvlishvili 1989; (6) Konstantinova-Antova and Tsvetkov 1988; (7) Present work, see Table 4; (8) Hojaev 1986; (9) Tsvetkov and Tsvetkova 1989; (10) Jankovics 1975; (11) Haro et al. 1976; (12) Mirzoyan and Ohanian 1986; (13) Parsamian et al. 1985; (14) Pulakos 1976a; (15) Pulakos 1976b; (16) Pulakos 1977a; (17) Pulakos 1977b; (18) Erastova 1981; (19) Mirzoyan et al. 1968; (20) Parsamian 1982.

TABLE 2. Flare star surveys in southern fields.*

\begin{tabular}{l|ccc}
\hline \multicolumn{1}{c|}{ field } & $\begin{array}{c}\text { no. of } \\
\text { flare stars }\end{array}$ & $\begin{array}{c}\text { total observing } \\
\text { time }(\mathrm{h})\end{array}$ & references \\
\hline Sco-Oph & 10 & 118.5 & 1,2 \\
Coalsack & $3^{* *}$ & 27 & $3,4,5$ \\
R CrA & 2 & 9 & 6,7 \\
$o$ Vel & 1 & 5.5 & 8 \\
\hline total & 16 & 160 & \\
\hline
\end{tabular}

* without Orion association (M42/M43), which is included in Table 1.

** plus 140 suspected fast irregular variables with small amplitudes found by Andrews (1972) in the first automatic search for flare stars and irregular variables.

(1) Haro and Chavira 1974; (2) Sun and Tong 1988; (3) Andrews 1972; (4) Sanduleak 1968; (5) Sanduleak 1969; (6) Hardy and Mendoza 1968; (7) Duerbeck and Tsvetkov 1989; (8) MacConnell and Mermilliod 1984. 


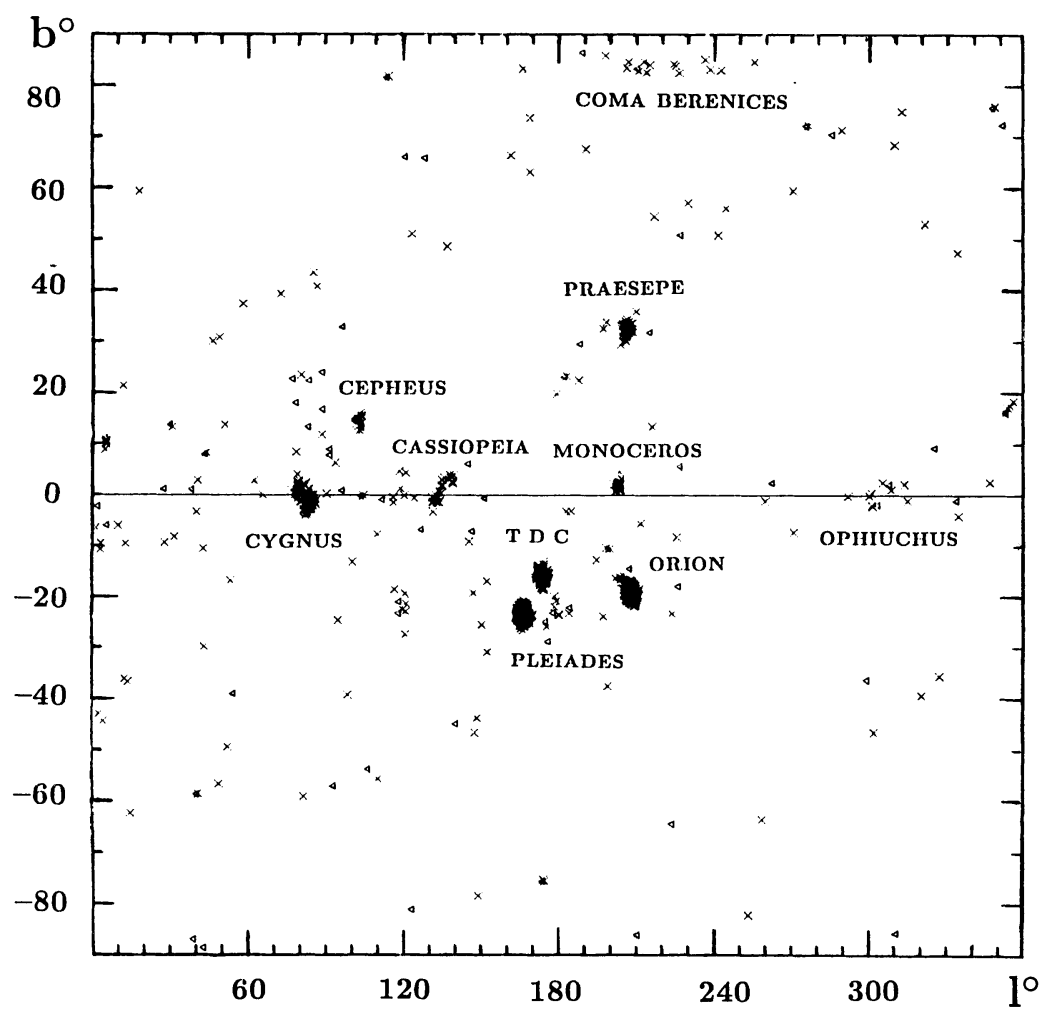

Fig. 1. Distribution of UV Ceti type stars in the Galaxy.

of the astrograph images. The field of the Orion association (M42/M43) which is not only well accessible from both hemispheres, but also one of the richest known regions of UV Cet and T Tauri stars, was chosen for tests of the detection power of the GPO and our reduction algorithms. The tests proved to be successful (Tsvetkov et al. 1986, Aniol et al. $1989)$.

\section{Observations}

The GPO astrograph has the following properties: $0.4 \mathrm{~m}$ aperture, focal ratio f:10, field size 4.2 square degrees, of those 2.3 square degrees practically coma-free. The complete fields are analyzed. With $10 \mathrm{~min}$ exposure time photographic magnitude 17.6 is reached on unsensitized Kodak IIa-O plates. Observations were carried out in the multiple exposure mode, usually with 5 to 7 observations per chain and ten minutes exposure time per image. To facilitate identifications of weak objects, deep plates were taken in the blue and red pass bands. So far, observations have been carried out during six observing periods in the southern summer and five observing periods in the winter; more observing time has been 
granted. The fields and the total monitoring times are listed in Table 3. The number of chains recorded per plate is of the order 10000 . The total number of stellar images on the plates collected so far is of the order 20 million. Thus, automated data analysis becomes a necessity, while offering the further advantages of objectivity, higher ability for measuring fainter flares and flare stars, and detectability of longer period light variations through plate to plate comparison of chains.

TABLE 3. Data for southern stellar aggregates.

\begin{tabular}{|c|c|c|c|c|c|c|c|c|c|}
\hline aggregate & $\begin{array}{c}\text { R A } \\
(1950)\end{array}$ & $\begin{array}{c}\text { Dec } \\
(1950)\end{array}$ & $l$ & $b$ & $\begin{array}{l}\text { age } \\
\text { (years) }\end{array}$ & $\begin{array}{l}\text { dist. } \\
\text { (pc) }\end{array}$ & $\begin{array}{l}\text { no. of } \\
\text { plates }\end{array}$ & $\begin{array}{l}\text { no. of } \\
\text { exp. }\end{array}$ & $\begin{array}{l}\text { obs. } \\
\text { time }\end{array}$ \\
\hline Orion $\mathrm{M} 42 / \mathrm{M} 43$ & $5^{\mathrm{h}} 33^{\mathrm{m}}$ & $-5^{\circ} 25^{\prime}$ & $209: 2$ & $-19^{\circ} .4$ & $5 \times 10^{5}$ & 460 & 52 & 326 & $57^{\mathrm{h}} 40^{\mathrm{m}}$ \\
\hline$o$ Vel & 540 & -5251 & 269.1 & -5.7 & $3 \times 10^{7}$ & 153 & 52 & 309 & $\begin{array}{ll}51 & 33\end{array}$ \\
\hline$\Theta \mathrm{Car}$ & 1042 & -6420 & 289.5 & -4.4 & $3 \times 10^{7}$ & 155 & 51 & 302 & 5020 \\
\hline Cha T1 & 1105 & -7720 & 297.0 & -15.3 & $\approx 10^{6}$ & 140 & 49 & 303 & 5210 \\
\hline Coalsack & 1149 & -6235 & 303.1 & 1.2 & $\approx 10^{6}$ & 174 & 21 & 126 & 2150 \\
\hline B228 Lup & 1249 & -3738 & 340.4 & 16.5 & $\approx 10^{6}$ & $150:$ & 38 & 227 & 3750 \\
\hline Sco-Oph & 1625 & -2410 & 353.9 & 17.1 & $\approx 10^{6}$ & 150 & 37 & 226 & 3740 \\
\hline B 59 Oph & 1712 & -2743 & 358.5 & 7.1 & $\approx 10^{6}$ & 250 & 41 & 231 & 3616 \\
\hline R CrA & 1901 & -7254 & 000.2 & -18.0 & $10^{7}$ & 129 & 53 & 228 & 5300 \\
\hline
\end{tabular}

\section{Measurement and Automatic Data Reduction}

Two flow diagrams of the program package FLARE, one for data preparation and one for data reduction, summarize the steps leading from photographic images to candidate lists of flare stars and other variables. The flow diagrams are shown in Figs. $2 a$ and $b$.

Digitization. In order to obtain the data in machine processible form, the plates are scanned with the PDS $2020 \mathrm{GM}^{\text {plus }}$ microdensitometer of the Muenster Astronomical Institute. With $20 \mu \mathrm{m}$ aperture and the same step size the relative positional accuracies are $0{ }^{\prime \prime} .07$ to $0 " 14$, depending on stellar brightness. Relative magnitudes within a given chain differ by no more than $0^{\mathrm{m}} .08$ to $0^{\mathrm{m}} .12$, depending on brightness. The total scan time per plate is $4^{\mathrm{h}}$, supervised time (plate adjustment, initialization) is less than $0.3^{\mathrm{h}}$. Up to 19 plates are stored on a single $8 \mathrm{~mm}$ video tape.

Segmentation. For storage, the digitized plate data are 'segmented', i.e., all images are stored in 'picture frames' of 15 pixel $\times 15$ pixel each. This mode assures a minimum of storage space ( $1 / 3$ that of a complete plate) and fast accessibility of all relevant information.

Image processing. This step includes the following procedures: Image reconstruction = object detection, determination of mean background density (averaged over several scan lines), local plate noise, positions in $x$ and $y$, object radii, second order moments, and machine magnitudes for all objects.

Catalogue of image data. Through calibration with external catalogues, positions in $\alpha, \delta$, and photographic magnitudes are determined. The input catalogue for astrometric data is 


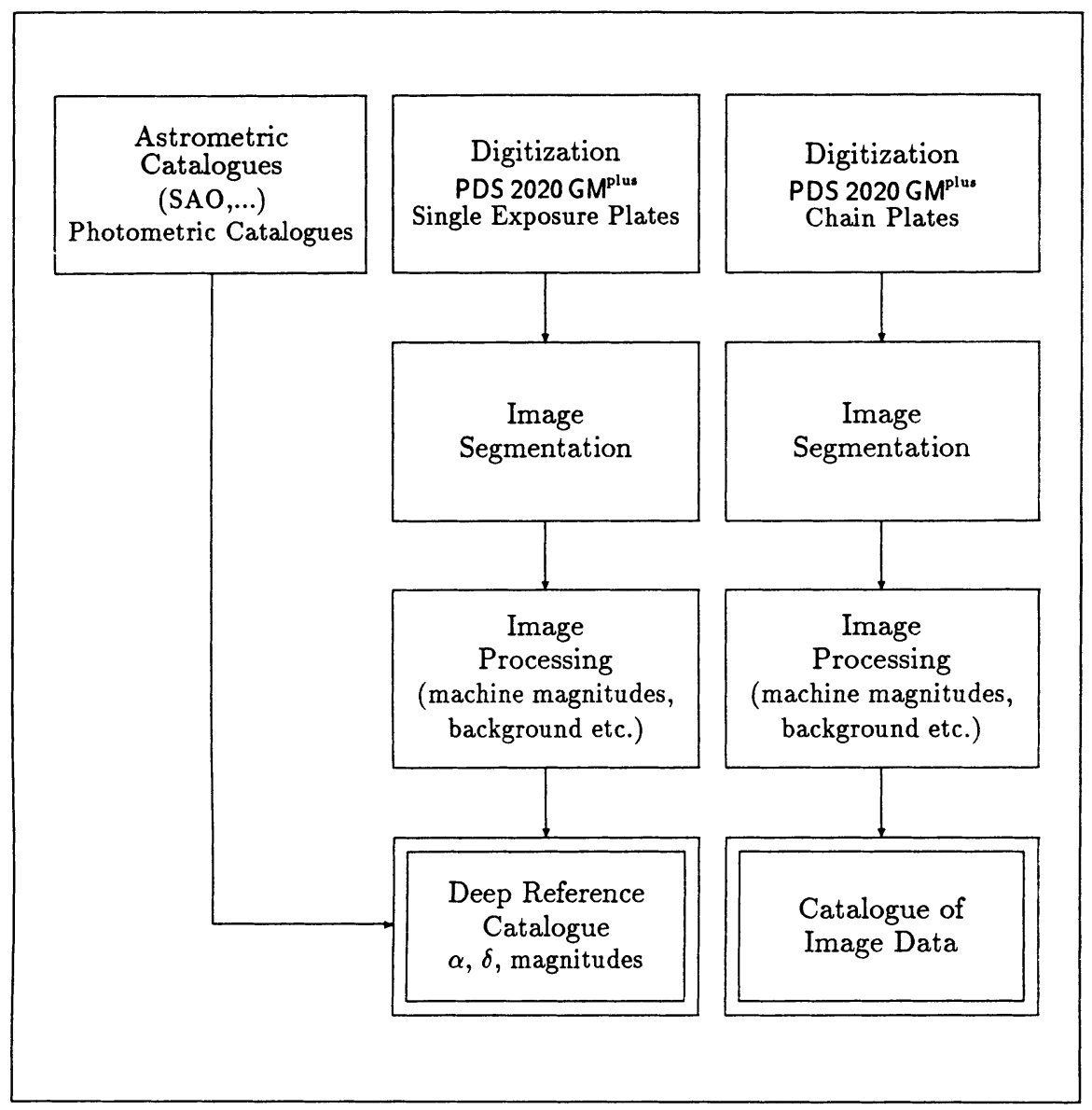

Fig. 2a. Preparation.

the SAO Catalogue, photometric data are taken from various sources. Catalogue positions are determined with an accuracy of $0^{\prime \prime} .5$. External magnitude calibrations are strongly affected by the lack of suitable standards. Their accuracy is at present $0{ }^{\mathrm{m}} \cdot 3$ to $0{ }^{\mathrm{m}} .4$.

Deep reference catalogue. The catalogue contains the machine and calibrated positions and magnitudes of all objects found on the deep plates. It is used in the subsequent reduction process.

Chain recognition by matching. All image data files are matched with the deep reference catalogue using the eastern images of the brightest chains for $x, y$-coordinate transformation. Positional accuracies after matching are 0"3. Also determined are the number of images per chain and the distances between chain images. The celestial coordinates from the deep plates are then assigned to all chains, including incomplete ones. The resulting lists of 


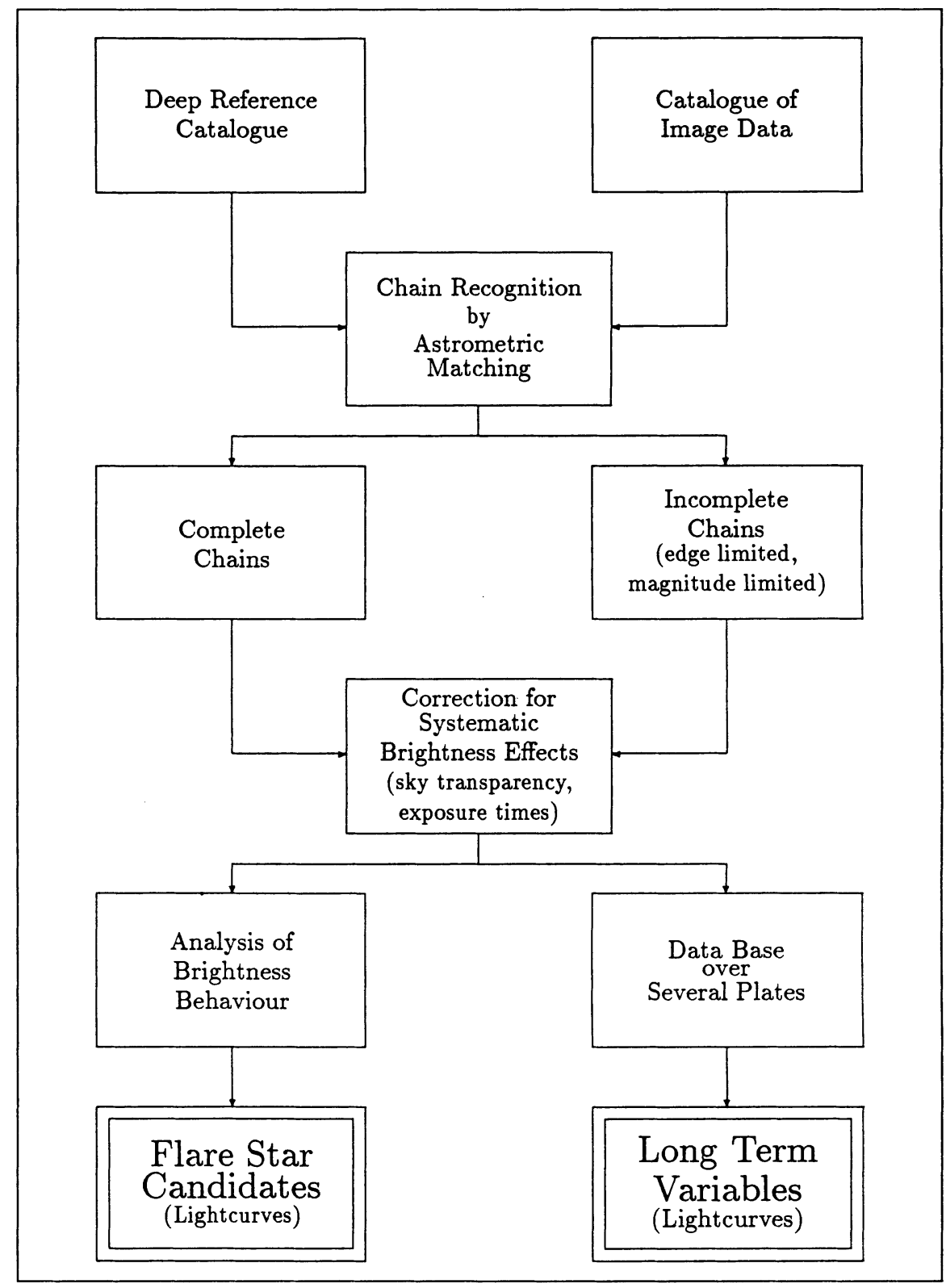

Fig. 2b. Main reduction. 
complete and incomplete chains are used for further processing.

Correction for systematic brightness effects. Statistical analyses of image brightnesses (machine magnitudes) are used to correct for differences in exposure time and sky transparency within the chains.

Analysis of brightness behaviour. From the corrected magnitudes 'light curves' for all chains of a given plate are determined. In order to avoid magnitude effects, mean light curves for different magnitude intervals (generally 6 intervals over the total magnitude range) are obtained.

Flare star candidates. By comparison of all individual chains on a plate with the mean light curve of the proper magnitude range, the stars whose chains include at least one image brighter than a preset limit are classified as flare star candidates. Because relatively high accuracy internal plate magnitudes are used, the limit can be set as low as $0^{\mathbf{m}} \cdot \mathbf{3}$ (approximately $3 \sigma$ ). In true flares, generally more than one image deviates from the mean. High resolution tracings and two-dimensional images of the chains of all candidates are checked visually, and final assignments as flares take into account the shapes of the observed outbursts.

Data base for all plates. All corrected chains found on all plates for a given star are collected in a data base.

Long term variables. This list contains all stars with light variations larger than the preset limit in any of the available chains, including those which have no identified flare event. For the determination of long term light variations the individual plate magnitudes are reduced to a common internal system. The accuracy is sufficiently high to detect variations within 0 . 5 . The majority of long term light curves found so far suggests irregular variables with time scales of days.

\section{Results from the test region and conclusions}

20 plates of the test region Orion M42/M43 were analyzed by the program FLARE. The results were compared with those obtained through visual inspection by one of us (M.K.T.) who has many years of experience in visual flare detection. The preset lower variation limit was $5 \sigma$ following Oskanian and Terebizh (1971). The results are summarized in Table 4, which also includes one new flare star found by visual inspection of a section not included in the above test. Samples of the detected flare events are shown in Fig. 3. From the candidates of the automatic reduction program, 12 flare events could be verified, the others were included in the list of long term variables. Visual inspection of the plates without preselection yielded 8 flares. Besides the fact that automatic search increased the number of objects by $50 \%$, it is interesting to note the characteristics of the additional flares: all are of low amplitude and some of them occurred on bright stars. They were verified by subsequent checks of the original plates. The results support our expectation that unbiased machine selection of flare candidates is particularly useful for objects which escape the strongly biased human eye. 10 of the observed stars are new flare stars. Six of them were previously known as variables (Kholopov 1985; Kholopov et al. 1985, 1987, 1989). This 

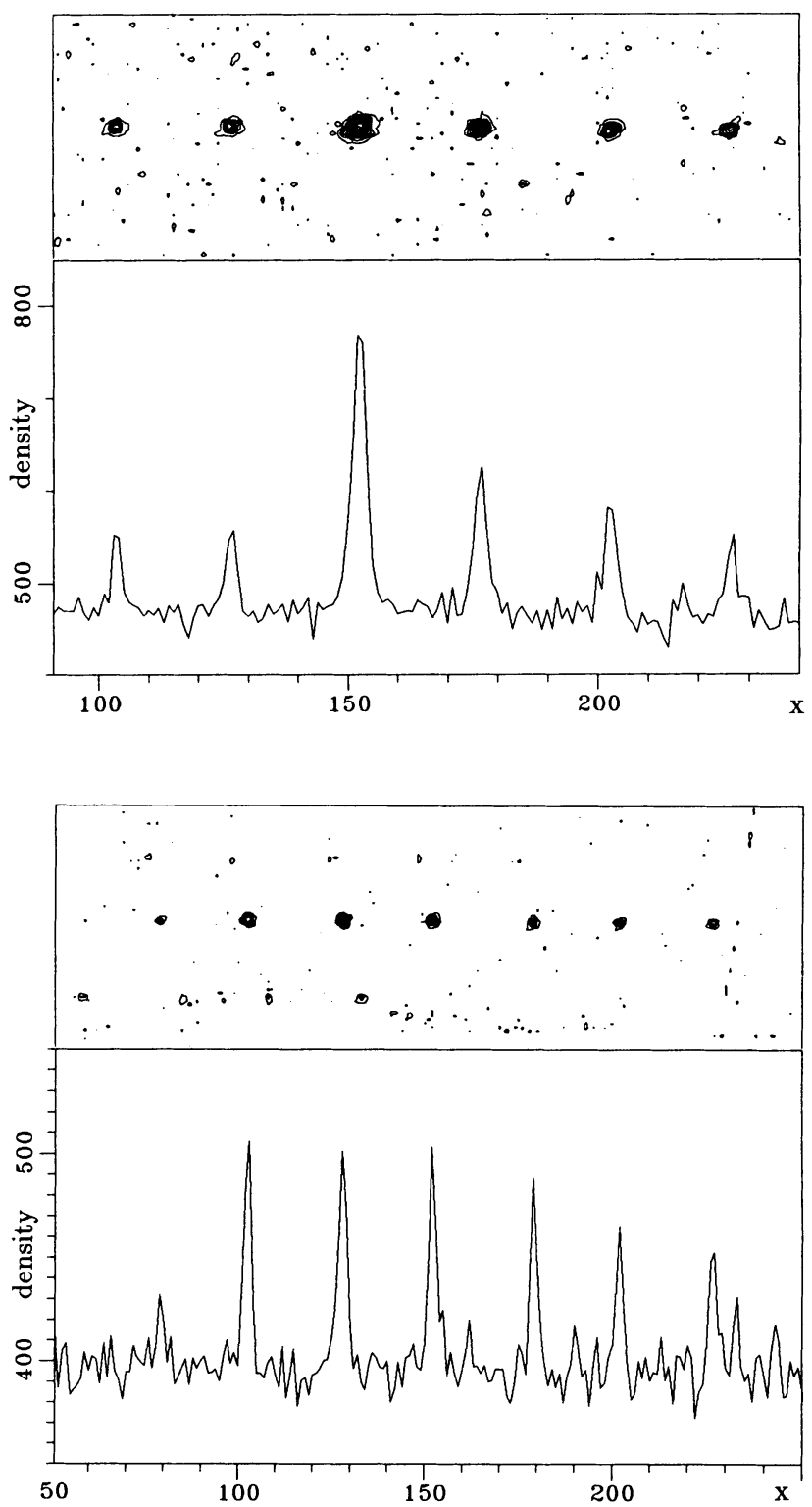

Fig. 3. Flare events in chains of LS6 and LS 7 shown as high resolution two-dimensional contour plots and density tracings. 
TABLE 4. New flare events and flare stars in the Orion M42/M43 association.

\begin{tabular}{|c|c|c|c|c|c|c|c|c|c|c|c|}
\hline $\begin{array}{l}\text { LS } \\
\text { no. }\end{array}$ & $\begin{array}{c}\mathrm{ABC} \\
\text { no. }\end{array}$ & $\begin{array}{c}\text { GCVS } \\
\text { CSV }\end{array}$ & & $\begin{array}{l}\text { A } \\
50)\end{array}$ & $\begin{array}{c}\text { Dec } \\
(1950)\end{array}$ & $\begin{array}{c}\text { date } \\
(1985)\end{array}$ & $\begin{array}{c}\mathrm{UT} \\
(\max )\end{array}$ & $\underset{\mathrm{pg}}{\min }$ & $\begin{array}{c}\max . \\
\text { pg }\end{array}$ & $\begin{array}{c}\Delta \mathrm{m} \\
(\mathrm{mag})\end{array}$ & notes \\
\hline 1 & & AR & $5^{h} 33$ & $\mathrm{n}_{28} \mathrm{~s}_{4}$ & $-5^{\circ} 06^{\prime} 59^{\prime \prime} 3$ & $10 \mathrm{Jan}$ & $2^{\mathrm{h}} 43^{\mathrm{m}}$ & $15^{\mathrm{m}} \cdot 16$ & $14^{\mathrm{m}_{53}}$ & 0.63 & 1 \\
\hline 2 & & V368 & 535 & 07.9 & $\begin{array}{llll}-5 & 13 & 45.1\end{array}$ & $11 \mathrm{Jan}$ & 126 & 15.84 & 15.20 & 0.65 & \\
\hline 3 & 97 & V714 & 530 & 45.9 & $\begin{array}{lll}-5 & 02 & 30.2\end{array}$ & $11 \mathrm{Jan}$ & 139 & 16.74 & 16.14 & 0.70 & 2 \\
\hline $4^{*}$ & 416 & & 532 & 22.7 & $\begin{array}{lll}-6 & 16 & 22.5\end{array}$ & $11 \mathrm{Jan}$ & 223 & $21.0:$ & 16.5 & 4.5: & \\
\hline 5 & 344 & 6315 & 536 & 13.5 & $\begin{array}{lll}-5 & 50 & 39.7\end{array}$ & $13 \mathrm{Jan}$ & $\begin{array}{ll}402 \\
0\end{array}$ & 16.92 & 16.32 & 0.59 & 3 \\
\hline 6 & 145 & & 534 & 02.4 & -44250.5 & $14 \mathrm{Jan}$ & 203 & 16.75 & 15.60 & 1.35 & 4 \\
\hline 7 & 172 & & 534 & 29.8 & -50045.8 & $15 \mathrm{Jan}$ & 142 & 16.89 & 16.07 & 0.82 & 3 \\
\hline 8 & & $\mathrm{AL}$ & 532 & 07.8 & $\begin{array}{lll}-5 & 46 & 39.9\end{array}$ & $17 \mathrm{Jan}$ & 238 & 16.11 & 15. 28 & 0.83 & \\
\hline 9 & & & 535 & 34.1 & -45456.9 & $21 \mathrm{Jan}$ & 153 & 16.84 & 15.79 & 1.15 & \\
\hline 10 & 52 & & $\begin{array}{ll}5 & 29\end{array}$ & 01.5 & -53943.3 & $26 \mathrm{Jan}$ & 245 & 16.46 & 15.34 & 1.12 & 5 \\
\hline 11 & 450 & & 536 & 11.1 & $\begin{array}{lll}-5 & 43 & 20.1\end{array}$ & $26 \mathrm{Jan}$ & 256 & 16.83 & 16.23 & 0.60 & \\
\hline 12 & 453 & & 536 & 23.9 & -52654.2 & $26 \mathrm{Jan}$ & $\begin{array}{ll}3 & 18\end{array}$ & 19.0 : & 16.32 & 1.7: & \\
\hline 13 & 239 & KO & 535 & 00.4 & -43305.8 & $26 \mathrm{Jan}$ & $\begin{array}{ll}329\end{array}$ & 15.84 & 14.24 & 1.65 & 1,3 \\
\hline
\end{tabular}

* Found in a section outside the automatically reduced field.

(1) slow flare; (2) discovered as flare star T15 by Haro (1953); (3) $\mathrm{H} \alpha$ emission star according to Parsamian and Chavira (1982); (4) discovered on patrol plate with exposures of 20 min per image; (5) discovered as flare star T260 by Haro and Chavira (1969).

$2 \%$ increase in the total number of known flare stars in Orion corresponds to a discovery rate of 0.5 per monitoring hour as compared to 0.3 for the same region from all previous surveys - not taking into account different field sizes and centers, and the pitfalls of small number statistics.

\section{Acknowledgements}

We thank the European Southern Observatory for observing time and assistance. Some of the reduction routines were kindly provided by H. Horstmann. M.K.T. acknowledges support from the Alexander von Humboldt Foundation. We also acknowledge travel grants from the International Astronomical Union (R.A.) and the Deutsche Forschungsgemeinschaft (W.S.).

\section{References}

Aniol, R., Duerbeck, H. W., Seitter, W. C. and Tsvetkov, M. K. (1988), Messenger 52, 39. Ambartsumian, V.A. (1969), Stars, Nebulae, Galaxies, Byurakan Symposium, Acad. Sci. Armenian SSR, Yerevan, p. 283.

Andrews, A. R. (1972), Bol. Obs. Tonantzintla y Tacubaya 6, No.38, 179.

Chavushian, H. S. (1979), Thesis, Yerevan.

Duerbeck, H.W., and Tsvetkov, M.K. (1989), in Mirzoyan, L.V., Pettersen, B. R., and Tsvetkov, M.K. (eds.) (1989), Flare Stars in Star Clusters, Associations and Solar Vicinity, IAU Symposium 137 (to be published). 
Erastova, L. K. (1981), Thesis, Yerevan.

Hardy, E., and Mendoza, E. E. (1968), Cordoba Bull. 14, 28.

Haro, G. (1953), Astrophys. J. 117, 513.

Haro, G., and Chavira, E. (1969), Bol. Obs. Tonantzintla y Tacubaya 5, No. 32, 59.

Haro, G., and Chavira, E. (1974), Bol. Inst. Tonantzintla 1, No. 3, 189.

Haro, G., Chavira, E., and Gonzales, G. (1976), Bol. Inst. Tonantzintla 2, No. 2, 95.

Haro, G., Chavira, E., and Gonzales, G. (1982), Bol. Inst. Tonantzintla 3, No. 1, 3.

Hojaev, A.S. (1986), in Mirzoyan, L. V. (ed.), Flare Stars and Related Objects, Acad. Sci. Armenian SSR, Yerevan.

Jankovics, I. (1975), Thesis, Yerevan.

Kholopov, P.N. (ed.) (1985), General Catalogue of Variable Stars, Nauka, Moscow.

Kholopov, P. N., Samus, N. N., Kazarovets, E. V., and Perova, N. B. (1985), Inf. Bull. Var. Stars No. 2681.

Kholopov, P.N., Samus, N.N., Kazarovets, E.V., and Kireeva, N. N. (1987), Inf. Bull. Var. Stars No. 3058.

Kholopov, P. N., Samus, N. N., Kazarovets, E. V., Florov, M.S., and Kireeva, N. N. (1989), Inf. Bull. Var. Stars No. 3032.

Konstantinova-Antova, R., and Tsvetkov, M. K. (1988), Inf. Bull. Var. Stars No. 3190.

MacConnell, D. J., and Mermilliod, J.-C. (1984), Inf. Bull. Var. Stars No. 2633.

Mirzoyan, L.V., Parsamian, E.S. and Chavushian, H.S. (1968), Soob. Byurakan Obs. 39, 2.

Mirzoyan, L. V., and Ohanian, G. B. (1986), in L. V. Mirzoyan (ed.), Flare Stars and Related Objects, Acad. Sci. Armenian SSR, Yerevan.

Natsvlishvili, R. Sh. (1989), A Catalogue of Flare Stars in the Orion T2 Association (to be published).

Oskanian, V.S., and Terebizh, V. Yu. (1971), Astrofizika 7, 281.

Parsamian, E.S. (1982), Thesis, Yerevan, 1982.

Parsamian, E. S., and Chavira, E. (1982), Bol. Inst. Tonantzintla 3, No.1, 69.

Parsamian, E.S., Rosino, L., and Chavushian, H.S. (1985), Astrofizika 22, 515.

Pulakos, C. (1976a), Praktika Acad. Athen 51, 765.

Pulakos, C. (1976b), Praktika Acad. Athen 51, 771.

Pulakos, C. (1977a), Astron. Astrophys. Suppl. 27, 429.

Pulakos, C. (1977b), Acta Astr. 27, 87.

Sanduleak, N. (1968), Inf. Bull. Var. Stars No. 275.

Sanduleak, N. (1969), Astrophys. J. 155, 1121.

Sun, Y.-l., and Tong, J.-h. (1988), Vistas Astr. 31, 385.

Tsvetkov, M. K., Seitter W.C., and Duerbeck, H.W. (1986), Comm. Konkoly Obs. No.86, 429.

Tsvetkov, M.K., and Tsvetkova, K.P. (1989), in Mirzoyan, L.V., Pettersen, B. R. and Tsvetkov, M.K. (eds.) Flare Stars in Star Clusters, Associations and Solar Vicinity, IAU Symposium 137 (to be published).

Tsvetkov, M. K., Stavrev, K. Y., and Tsvetkova, K. P. (1989), New machine-readable version of the Pleiades Flare Star Catalogue (in preparation). 